\title{
Neue südamerikanische Arten der Bienengattung Prosopis Fabr.
}

Von C. Schrottky (Puerto Bertoni, Paraguay).

Übersicht der nachstehend beschriebenen Arten.

1. Mittelsegment hinten gerundet.

- Mittelsegment hinten abgestutzt.

2. Pronotum und Scutellum gelb (Bolivien) 1. Pr. mapiriensis

- Pronotum und Scutellum schwarz (Brasil.). 2. brasiliensis

3. Clypeus größtenteils gelb, kleinere Arten.

- Clypeus ganz schwarz, große Art (Peru).

3. Bequaerti

4. Pronotum und Scutellum mit gelber Zeichnung. 5

- Pronotum und Scutellum ganz schwarz. 7

5. Nur das erste Abdominalsegment deutlich punktiert; Scutellum ohne gelbe Axillarflecken.

Die beiden ersten Segmente grob, aber nicht sehr dicht punktiert; Scutellum mit gelben Axillarflecken (Peru). 4. peruviana

6. Scutellum mit zwei gelben Querflecken (Brasil.) 5. stenops

- Scutellum ganz gelb (Bolivien). 6. sculptilis

7. Erstes Abdominalsegment deutlich punktiert (Bolivien).

\section{7. boliviana}

Abdomen ohne erkennbare Skulptur.

8. Mesonotum durch eine tiefe Längsfurche geteilt, mit Querrunzeln (Bolivien).

8. bothros

Mesonotum ohne tiefe Längsfurche, punktiert (Brasilien).

9. exigua

\section{Prosopis mapiriensis n. sp.}

O .Der Pros. scrobicauda Vach. nahestehend, aber durch ganz anders gebildetes Mittelsegment verschieden. Schwarz. Clypeus (mit Ausnahme der breiten schwarzbraunen Seitenränder), Nebengesicht, innere Augenränder bis drei Viertel ihrer Höhe, Scutum nasale und ein großer Doppelfleck zwischen den Fühlern gelb. Fühler rostfarben. Oberkopf äußerst fein und dicht punktiert. Am Thorax sind gelb: Pronotum, Schulterbeulen, ein Fleck auf den Tegulae und der größte Teil des Scutellum. Mesonotum mit äußerst dichter und feiner Punktierung, die der Pleuren fein und zerstreut. Mittelsegment hinten gerundet, ganz ohne wahrnehmbare Skulptur, dabei matt, nur 
in der Mitte des Basalteiles ein länglicher Höcker; an den Seiten ganz kurze weißliche Behaarung. An den Beinen sind die vorderen Schienen außen ganz, die übrigen an der Basis gelb gefärbt. Flügel glashell mit braunem Geäder; nerv. rec. 1 unmittelbar vor dem Ende der ersten Kubitalzelle. Erstes Abdominalsegment vollkommen poliert, ohne Spur von Punktierung; die übrigen matt und mit weißlichen Haarbinden auf den Apikalrändern. Läng e 41/2 mm, Abdomenbreite $1 \mathrm{~mm}$. - Bolivien, Dep. La Paz, Mapiri.

Durch Staudinger und Bang-Haas erhalten.

Das Gelb des Pronotum und Scutellum ist bei einigen Exemplaren reduziert, so daß im äußersten Falle am Pronotum nur die Ecken, am Scutellum nur die Mitte gelb bleiben. Untersucht wurden fünf Exemplare.

\section{Prosopis brasiliensis n. sp.}

ç Sehr ähnlich der Pros. paraguayensis m., aber durch den durchaus glatten Basalteil des Mittelsegmentes leicht zu unterscheiden. Schwarz. Gesicht bis zu den Fühlern und innere Augenränder darüber hinaus gelb. Fühler, einschließlich Schaft, unten bräunlich gelb, oben dunkelbraun. Oberkopf äußerst dicht punktiert. Am Thorax sind nur die Schulterbeulen und ein Fleckchen auf den Tegulae gelb. Die Punktierung des Mesonotum besteht aus dichten, aber äußerst feinen Runzeln. Die Pleuren sind glänzend und mit wenigen, bei 20 -facher Vergrößerung erst eben erkennbaren Pünktchen. Mittelsegment gerundet; Basalteil in der Mitte mit kurzem Kiel, jederseits davon glatt und glänzènd; der hintere Teil durch eine Furche geteilt. Schienen der Vorder- und Nittelbeine gelb, die der letzteren braun gefleckt, Schienen der Hinterbeine mit gelber Basis. Flügel glashell, lebhaft irisierend, Geäder hellbraun; nerv. rec. 1 interstitial. Abdomen mit ganz feiner, eben erkennbarer Punktierung auf den beiden ersten Segmenten, das erste ist stark glänzend, das zweite, wie die folgenden eher matt; an den Seiten aller Segmente ein wenig weißliche Behaarung (keine Binden!). Länge $4 \mathrm{~mm}$. Abdomenbreite $0.7 \mathrm{~mm}$.

Brasilien, Staat S. Paulo, Piracicaba. XI. 06.

Die Type ist Eigentum des Museu Paulista.

\section{Prosopis Bequaerti n. sp.}

Q Eine ganz isoliert stehende Art, welche leicht an der ungewöhnlichen Größe und dem einfarbig schwarzen Clypeus erkannt werden kann. Ganz schwarz, nur Nebengesicht und innere Augen- 
ränder gelb; an den Beinen ist nur die Basis der vordersten Schienen gelblich. Clypeus und Scutum nasale fein nadelrissig längsgerieft. Oberkopf äußerst dicht und fein runzelig punktiert. Fühler schwarz. Mesonotum matt, mit feiner, seichter, zerstreuter Punktierung. Die Pleuren sind äuferst fein körnelig, dazwischen mit zerstreuter deutlicher Punktierung. Scutellum und Metanotum wie das Mesonotum. Mittelsegment hinten abgestutzt, der Basalteil grob netzartig gerunzelt. Schulterbeulen außen mit einem Kranze weißlicher Haare umgeben. Flügel rauchig getrübt, Tegulae und Geäder schwarzbraun; nerv. rec. 1 etwas vor dem Ende der ersten Kubitalzelle. Abdomen glatt und glänzend, die beiden ersten Segmente mit zerstreuter undeutlicher Punktierung, welche nur auf den Seiten des ersten deutlicher und auch dichter wird.

Länge $8 \mathrm{~mm}, \mathrm{Abdom}$ enbreite $2 \mathrm{~mm}$.

Perú, Chanchamayo. 700-1000 m. (1906).

Ein $q$ von Herrn Dr. Jos. Bequaert, Alost, erhalten und ihm freundschaftlichst gewidmet.

\section{Prosopis peruviana n. sp.}

$\delta^{7}$ Ich hielt mein einziges Exemplar anfangs für Pros. cribellata Vach.; es ergeben sich aber so viele Unterschiede, daß die Aufstellung einer besonderen Art gerechtfertigt ist. In der Färbung unterscheidet sich peruviana wie folgt: Die äuferen Augenränder sind nicht gelb gerandet, das Pronotum ist ganz gelb, nur in der Mitte schmal unterbrochen, am Scutellum sind nicht nur die Axillarflecke gelb, sondern auch zwei größere Querflecke auf der Scheibe des Scutellum selbst. Die beiden Basalglieder der Fühler sind vorne gelb; das Scutum nasale ist fast gleichseitig fünfeckig und ganz gelb. In der Skulptur weicht peruviana ab, indem die Furchen des Basalteiles des Mittelsegments nach hinten zu nicht konvergieren, eher das Gegenteil ; die beiden ersten Abdominalsegmente sind glänzend, dabei grob aber nicht sehr dicht punktiert; das vorletzte Segment ist im ausgereckten Zustande etwas rötlich. Die Flügel sind glashell; nerv. rec. 1 interstitial. Im übrigen wie cribellata. Läng e $5 \mathrm{~mm}$. Abdomenbreite $1 \mathrm{~mm}$. - Perú, Chanchamayo.

Von Herrn Dr. Jos. Bequaert, Alost, erhalten.

In der Beschreibung der $\mathrm{Pr}$. cribellata ist ein Fehler stehen geblieben. Erst heißt es: "mesonotum punctis crassis confluentibus", eine Zeile weiter: „mesonotum antice et postice marginatum, punctis sparsis“. Das zweite Mal soll es vermutlich „Mesosternum" heißen. 


\section{Prosopis stenops n. sp.}

ơ Gehört in die Nähe der Pros. peruviana m. Schwarz, Mund, Gesicht bis zu den Fühlern, innere Augenränder fast ganz und die beiden Basalglieder der Fühler vorn gelb; der Rest der Fühler ist vorn gelbbraun, oben braun. Das gelbe Scutum nasale reicht mit der Spitze bis zwischen die Fühler. Oberkopf ziemlich grob und sehr dicht punktiert. Am Thorax sind gelb: Das Pronotum (ausgenommen in der Mitte), die Schulterbeulen, ein Fleck auf den Tegulae und zwei Querflecke auf dem Scutellum. Mesonotum ziemlich grob, aber nicht sehr dicht punktiert, desgleichen die Pleuren. Mittelsegment hinten abgestutzt; Basalteil hinten halbkreisförmig ausgerandet, mit zwei nach hinten zu etwas konvergierenden Längskielen, jederseits davon mehrere strahlige Runzeln. Schienen der Vorder- und Mittelbeine außen ganz gelb, die der Hinterbeine mit gelber Basis. Flügel glashell, lebhaft irisierend, Geäder braun, beide rücklaufende Nerven interstitial oder fast so. Abdomen mit spärlich punktiertem ersten Tergit, das zweite mit kaum noch unterscheidbarer feiner Punktierung; beide sowie das dritte auf den Apikalrändern mit weißen feinen Haarbinden, zum mindesten auf den Seiten. Länge $4 \frac{1}{2} \mathrm{~mm}$, Abdomenbreite kaum $1 \mathrm{~mm}$.

Brasilien, Staat S. Paulo, Piracicaba XI. 06.

Die Type ist Eigentum des Museu Paulista.

\section{Prosopis sculptilis n. sp.}

O In Färbung und Größe der Pros. gracillima m. sehr ähnlich, aber durch die stärkere Skulptur, namentlich des ersten Abdominalsegments, verschieden; von Pros. aspricollis Vach. unterscheidet sich die neue Art durch die Größe, Herkunft und gleichfalls durch stärkere Skulptur des ersten Segments. Schwarz. Clypeus (mit Ausnahme der breiten, tief braunen Seitenränder), Scutum nasale, ein Doppelfleckchen zwischen den Fühlern, Nebengesicht und innere Augenränder gelb, Fühler rostbraun, Geißel oben dunkel braun. Stirn mit deutlichem Längskiele bis zum vorderen Ocellus. Kopf im allgemeinen sehr dicht und fein punktiert. Am Thorax sind gelb: Pronotum, Schulterbeulen, ein Fleck auf den Tegulae und Scutellum. Mesonotum sehr dicht und mäßig grob punktiert, schwach goldig schimmernd. Pleuren sehr grob und mäßig dicht punktiert. Scutellum glänzend, mit zahlreichen ziemlich groben Punkten. Metanotum dicht und fein punktiert. Basalteil des Mittelsegments mit fünf kurzen Längskielen; Rest weißlich behaart. Tibien der Vorder- 
beine außen ganz gelb, die der übrigen mit gelber Basis. Flügel glashell mit braunem Geäder; nerv, rec. 1 interstitial. Erstes Abdominalsegment glänzend mit grober nicht sehr dichter Punktierung; der Rest matt, ohne deutliche Skulptur; Anus kurz schwarz behaart. Länge $6 \mathrm{~mm}$; Abdomenbreite $1.7 \mathrm{~mm}$.

Bolivien, Dep. La Paz, Mapiri.

Diese Art ist ziemlich variabel: zunächst können sämtliche Segmente am Apex weiße Haarbinden haben, oder diese sind ganz oder teilweise abgerieben; ferner können alle oder ein Teil der fünf Längskiele des Mittelsegments doppelt auftreten, so daß ihre Zahl bis 10 steigt. Die Punktierung des ersten Segments greift häufig auf das zweite über und schließlich fehlt häufig der Doppelfleck zwischen den Fühlern. Von den sieben untersuchten Exemplaren gleichen sich nicht zwei ganz vollkommen.

\section{Prosopis boliviana n. sp.}

q Dürfte in die Nähe von Pros. palmaris Vach. gehören, von welcher nur das $\delta^{7}$ bekannt ist, aber boliviana hat das erste Abdominalsegment deutlich punktiert, ist größer etc. Schwarz. Clypeus (mit Ausnahme der dunkelbraunen Seitenränder), Nebengesicht, innere Augenränder und Scutum nasale gelb. Fühler braun mit rostfarbenen Basalgliedern. Oberkopf gerundet, sehr dicht und ziemlich grob punktiert. Am Thorax sind nur die Schulterbeulen und ein Fleck auf den Tegulae gelb, Pronotum etwas länger als gewöhnlich, glatt, scharf gerandet mit vorspringenden Ecken. Mesonotum mit äußerst grober, in der Vorderhälfte desselben mit zusammenfließender Punktierung. Scutellum matt, mit wenigen groben Punkten. Pleuren grob, aber nicht sehr dicht punktiert. Mittelsegment hinten abgestutzt, ebenda weißlich behaart, Basalteil von unregelmäßigen Längskielen durchzogen. Schienen der Vorderbeine aufen ganz, die übrigen nur an der Basis gelb. Flügel glashell mit braunem Geäder; nerv. rec. 1 interstitial. Abdominalsegment 1 deutlich punktiert; die übrigen matt, mit weißen Haarbinden auf den Apikalrändern. L än g e $5^{1 / 2} \mathrm{~mm}$; Abdomenbreite $12 \mathrm{~mm}$. - Bolivien, Dep. La Paz, Mapiri.

Durch Staudinger \& Bang-Haas erhalten.

Untersucht wurden 3 Exemplare.

\section{Prosopis bothros n. sp.}

+ Der Pros. paradoxa m. sehr nahe stehende Art mit gleichfalls tiefer Längsgrube auf dem Mittelrücken; Färbung und Skulptur 
des Mittelsegments dagegen sehr verschieden. Clypeus (mit Ausnahme der Seitenränder), Nebengesicht und innere Augenränder gelb, Mandibel und Fühler braun, Schaft dunkler. Scheitel dicht und mäßig grob punktiert; Hinterhauptsrand ziemlich scharf. Am Thorax sind nur die Schulterbeulen sowie ein Fleckchen auf dem Außenrande der Tegulae gelb. Mesonotum seiner ganzen Länge nach durch eine tiefe Längsgrube geteilt, jederseits davon mit einigen Querrunzeln, nach den Seiten zu matt und vereinzelt punktiert. Parapsidien ebenfalls tief. Scutellum und Metanotum matt, zerstreut punktiert. Mittelsegment mit ziemlich kurzem Basalfelde; dieses von unregelmäßigen, kurzen, gezackten Kielen durchzogen, hinten scharf abgesetzt. Beine mit gelb gezeichneten Schienen, und zwar sind die vordersten außen ganz, die übrigen nur an der Basis gelb. Flügel glashell mit braunem Geäder, nerv. rec. 1 dicht vor dem Ende der ersten Kubitalzelle. Abdomen ohne erkennbare Skulptur. Länge $5 \mathrm{~mm}, \mathrm{Abd}$ om en breite $1.2 \mathrm{~mm}$. - Bolivien, Dep. La Paz, Mapiri.

Abweichungen: Der Vorderrand des Scutum nasale zeigt häufig Neigung gelb zu werden. Selten ist der nerv. rec. 1 interstitial.

Untersucht wurden 21 Exemplare, der Firma Dr. O. Staudinger u. A. Bang-Haas gehörig.

\section{Prosopis exigua Schrottky.}

Das $\sigma^{7}$ ist in „Revista do Museu Paulista. Vol. V., 1902, p. 341" beschrieben. Das \& war noch nicht bekannt: \& Schwarz. Clypeus (mit Ausnahme der Seitenränder), Scutum nasale, Nebengesicht und innere Augenränder bis fast zum Scheitel gelb. Fühler vorn hellbraun, oben dunkler. Oberkopf sehr fein und dicht körnig punktiert. Mesonotum matt, mit tiefen, eingestochenen, länglichen Punkten; diese sind in der Mitte gröber und weniger dicht als an den Rändern. Schulterbeulen gelb. Pleuren matt, mit äußerst feinen und sehr zerstreuten Pünktchen. Scutellum zerstreuter punktiert als Mesonotum. Mittelsegment hinten abgestutzt; Basalteil rings gerandet, in der Mitte mit V-förmigen Leisten, jederseits davon einige strahlige Runzeln. Schienen der Vorderbeine gelb, die der übrigen mit gelber Basis. Flügel glashell, Geäder braun; nerv. rec. 1 interstitial, nerv. rec. 2 kurz vor dem Ende der zweiten Kubitalzelle. Abdomen ohne wahrnehmbare Skulptur. Länge $5 \mathrm{~mm}, \mathrm{Abdom}$ enbreite $1.3 \mathrm{~mm}$.

Brasilien, Staat S. Paulo, Piracicaba XI. 06.

Die Type ist Eigentum des Museu Paulista. 


\section{$2 \mathrm{BHL}$ Biodiversity Heritage Library}

Schrottky, Curt. 1910. "Neue südamerikanische Arten der Bienengattung Prosopis FABR." Wiener entomologische Zeitung 29, 133-138.

https://doi.org/10.5962/bhl.part.23340.

View This Item Online: https://www.biodiversitylibrary.org/item/44117

DOI: https://doi.org/10.5962/bhl.part.23340

Permalink: https://www.biodiversitylibrary.org/partpdf/23340

\section{Holding Institution}

Smithsonian Libraries

\section{Sponsored by}

Smithsonian

\section{Copyright \& Reuse}

Copyright Status: NOT_IN_COPYRIGHT

This document was created from content at the Biodiversity Heritage Library, the world's largest open access digital library for biodiversity literature and archives. Visit BHL at https://www.biodiversitylibrary.org. 